\title{
Decoherence and relaxation of a single electron in a one dimensional conductor
}

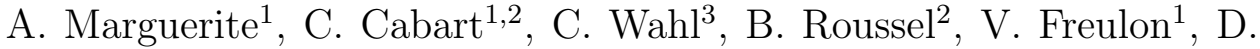 \\ Ferraro $^{2,3}$, Ch. Grenier ${ }^{2}$, J.-M. Berroir ${ }^{1}$, B. Plaçais ${ }^{1}$, T. Jonckheere ${ }^{3}$, J.

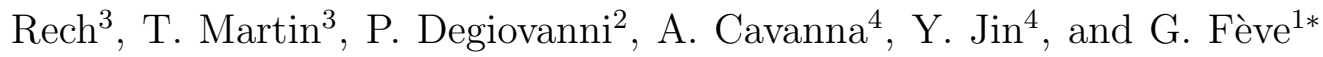 \\ ${ }^{1}$ Laboratoire Pierre Aigrain, Ecole Normale Supérieure-PSL Research University, \\ CNRS, Université Pierre et Marie Curie-Sorbonne Universités, Université Paris \\ Diderot-Sorbonne Paris Cité, 24 rue Lhomond, 75231 Paris Cedex 05, France \\ 2 Univ Lyon, ENS de Lyon, Univ Claude Bernard, \\ CNRS, Laboratoire de Physique, F-69342 Lyon, France \\ 46 Allée d'Italie, 69364 Lyon Cedex 07,France. \\ 3 Aix Marseille Université, Université de Toulon, \\ CNRS, CPT, UMR 7332, 13288 Marseille, France \\ ${ }^{4}$ Laboratoire de Photonique et Nanostructures (LPN) \\ CNRS, Université Paris-Saclay, Route de Nozay, 91460 Marcoussis, France \\ * To whom correspondence should be addressed; E-mail: feve@lpa.ens.fr.
}

\begin{abstract}
We study the decoherence and relaxation of a single elementary electronic excitation propagating in a one-dimensional chiral conductor. Using two-particle interferences in the electronic analog of the Hong-Ou-Mandel experiment, we analyze quantitatively the decoherence scenario of a single electron propagating along a quantum Hall edge channel at filling factor 2 . The decoherence results from the emergence of collective neutral excitations induced by Coulomb interaction and leading, in one dimension, to the destruction of the elementary quasiparticle. This study establishes the relevance of electron quantum optics setups to provide stringent tests of strong interaction effects in one-dimensional conductors described by Luttinger liquids paradigm.
\end{abstract}




\section{INTRODUCTION}

What is the fate of a single electron propagating in a conductor? This basic problem of condensed matter physics has given birth to the Landau-Fermi liquid paradigm in 3D solids: Coulomb interactions limit the phase coherence at low temperatures [1] but nevertheless do not invalidate the single electron as a quasiparticle [2]. At the opposite in 1D systems, Coulomb interactions favor the emergence of collective modes [3, 4] leading to the disappearance of the single electron as a good quasiparticle [5, 6], thus giving rise to the Luttinger liquid paradigm for 1D quantum wires [7]. However, observing this transition from single to many body physics requires a more elaborate experimental scheme than a direct measurement of the current flowing in the conductor as charge propagation remains unaffected by interactions. Coulomb interactions have already been shown to be responsible for electronic decoherence [8-10] in single particle interferometers [11, 12] and for the relaxation of non-equilibrium energy distribution [13, 14]. However the fate of the single elementary quasiparticle could not be accessed as stationary sources were used, lacking both temporal dependence and single electron resolution. In this paper, we use two-particle interferences in the electronic analog [15, 16] of the Hong-Ou-Mandel (HOM)[17] experiment to analyze quantitatively the decoherence [18] of a single electron along its propagation within the outer edge channel of the integer quantum Hall effect at filling factor 2. The HOM experiment thus probes for the first time the decay of a single Landau quasiparticle in a ballistic conductor. By revealing the fate of a single electronic excitation and confirming our predictions for its decoherence scenario [6, 18, this study demonstrates how electron quantum optics techniques provide a powerful probe of strong interaction effects in ballistic conductors.

The characterization of a single electron state stems from the study of its coherence properties. The single electron coherence of an electron source at position $x$ of an edge channel [19, 20] can be defined using a quantum optics formalism [21]: $\mathcal{G}_{x}^{(e)}\left(t, t^{\prime}\right)=\left\langle\Psi^{\dagger}\left(x, t^{\prime}\right) \Psi(x, t)\right\rangle$, where the electric field of quantum optics has been replaced by the fermion field operator $\Psi(x, t)$ which annihilates an electron at time $t$ and position $x$ of the edge channel (as the position $x$ will be fixed, it will be dropped in the following). Most experiments investigating electron coherence have been performed using stationary sources (using a dc voltage

bias) which continuously emit electrons in the conductor. In the stationary case, $\mathcal{G}^{(e)}$ only depends on the time difference $\tilde{\tau}=t-t^{\prime}$ and provides information on the coherence time 
of the source, but does not depend on $\bar{t}=\left(t+t^{\prime}\right) / 2$. Single electron coherence is thus fully determined by the mere knowledge of the energy distribution of the emitted electrons (through Fourier transformation). The situation is very different when one uses the recently developed single electron emitters [22, 26], which trigger the emission of a single particle at a well defined time, such that the full $t, t^{\prime}$ dependence needs to be retained. For an ideal single electron source, $\mathcal{G}^{(e)}\left(t, t^{\prime}\right)$ fully encodes the emitted single electron wavepacket $\varphi_{e}(t)$ : $\Delta \mathcal{G}^{(e)}\left(t, t^{\prime}\right)=\mathcal{G}^{(e)}\left(t, t^{\prime}\right)-\mathcal{G}_{F}^{(e)}\left(t-t^{\prime}\right)=\varphi_{e}^{*}\left(t^{\prime}\right) \varphi_{e}(t)$, where $\mathcal{G}_{F}^{(e)}$ is the equilibrium contribution of the Fermi sea. Probing single electron coherence thus brings the possibility to picture single particle states propagating in solid state [19, 27] and characterize how single electron wavepackets evolve under the influence of Coulomb interaction. In this non-stationary situation, in the frequency domain, $\widetilde{\mathcal{G}}^{(e)}\left(\omega, \omega^{\prime}\right)$ has two Fourier components $\omega$ and $\omega^{\prime}$ [19]. The energy distribution, which has been recently measured [13, 14], only contains a partial information on single electron coherence (diagonal part $\omega=\omega^{\prime}$ ). The non-diagonal elements $\left(\omega \neq \omega^{\prime}\right)$ contain all the information on the non-stationary aspects of electron coherence $(\bar{t}$ dependence). As we shall see, their knowledge is thus essential for predicting HOM traces but also to predict the evolution of the non-stationary state under the influence of Coulomb interaction. In the presence of strong Coulomb interactions, this problem cannot be reduced to the redistribution of the electron energy distribution caused by Coulomb induced electron-electron scattering.

This paper is organized as follow. In Section II we discuss the properties of single electron coherence and how it can be efficiently probed in the Hong-Ou-Mandel experiment. Experimental results are presented in Section III. Single electron decoherence shows up in the electronic HOM experiment as a reduction of the two-particle interference contrast. These results are discussed using a phenomenological approach of electronic decoherence. In Section IV we present various possible mechanisms which could lead to the observed contrast reduction. By ruling out progressively the majority of them by a direct comparison with the experimental results, we can identify the inter-channel Coulomb interaction as the dominant source of contrast reduction. In Section V, we quantitatively compare our experimental observations with non-perturbative bosonization-based models for Coulomb interaction induced single electron decoherence at filling factor $\nu=2$. This allows us to properly fit the experimental HOM traces without the need to introduce any unnecessary phenomenological parameters. This agreement establishes the complete picture of the destruction scenario of 
a single electron in a chiral one-dimensional conductor.

\section{ELECTRON COHERENCE IN THE ELECTRONIC HONG-OU-MANDEL EX- PERIMENT}

In the electronic HOM experiment (see Fig,1, right panel), two quantum dots driven by step voltage pulses (with a repetition frequency $f=0.9 \mathrm{GHz}$ and a typical risetime of 30 picoseconds) are used as single electron sources [22]. The peak to peak voltage pulse amplitude matches the dot addition energy $\Delta=1.4 \mathrm{~K}$ such that a single electron followed by a single hole are emitted in the outer edge channel at energy $\omega_{e}=\omega_{h}=0.7 \mathrm{~K}$ above (electron) or below (hole) the Fermi level. The dot to edge channel transmission can be tuned to vary the escape time $\tau_{e}$ (and hence the wavepacket length) of the emitted electrons and holes. The electron sources are placed at $l=3 \mu \mathrm{m}$ upstream of the inputs 1 and 2 of a quantum point contact (QPC) used as an electronic beam-splitter.

When two electrons collide synchronously on the splitter, two paths (see Fig. 1, left panel) contribute to the coincidence detection of the detectors placed at outputs 3 and 4 : either a particle in input 1 is detected in output 3 and 2 goes to 4 (blue path) or 1 goes to 4 and 2 goes to 3 (red path). The sum of the two probability amplitudes for the corresponding exchanged paths leads to interferences involving two particles at the input [28 30]. These two-particle interferences can be revealed by the measurement of the low-frequency current correlations (or noise) at the output of the splitter $\Delta S_{33}$ (where $\Delta$ refers to the excess noise after subtraction of the equilibrium noise contribution), and used to provide information on the excess single electron coherence $\Delta \mathcal{G}_{i}^{(e)}$ of the sources placed at inputs $i=1,2$ [19]:

$$
\begin{aligned}
& \Delta S_{33}=\Delta S_{\mathrm{HBT}}-\Delta S_{\mathrm{HOM}} \\
& \Delta S_{\mathrm{HBT}}=2 R(1-R) e^{2} f \int_{0}^{\infty} d \epsilon\left(\delta n_{1}^{e / h}(\epsilon)+\delta n_{2}^{e / h}(\epsilon)\right)\left(1-2 f_{\mu, T}(\epsilon)\right)
\end{aligned}
$$

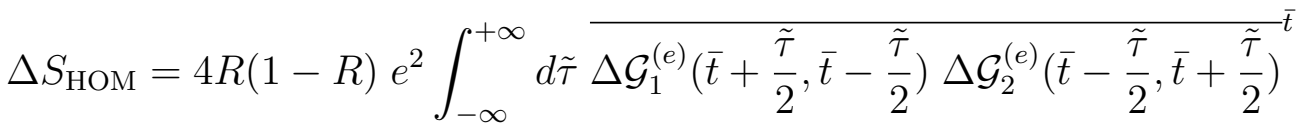

where $R$ is the reflection probability of the beam-splitter, $f_{\mu, T}(\epsilon)$ the Fermi distribution and $\delta n_{i}^{e / h}(\epsilon)$ the energy density of electron and hole excitations emitted by source $i$ in one period $1 / f$. The first term labeled $\Delta S_{\mathrm{HBT}}$ in (1) represents the random partitioning of

quasiparticles on the beam splitter. As can be seen from Eq.(2), at zero temperature it 
is directly proportional to the total number of excitations (electrons and holes) emitted by sources 1 and 2. At finite temperature, the partitioning of low energy electron/hole pairs is reduced by two-particle interferences with thermal Fermi sea excitations [31]. The second term labeled $\Delta S_{\mathrm{HOM}}$ in $(1)$ is the two-particle interference term. It comes with a minus sign, as a consequence of the fermionic statistics, and thus reduces the random partitioning. As can be seen from Eq.(3), it is given by the overlap between the single electron coherence of the two sources and thus probes both their diagonal $\left(t=t^{\prime}\right.$ or $\omega=\omega^{\prime}$ in Fourier space) as well as off-diagonal $\left(t \neq t^{\prime}\right.$ or $\left.\omega \neq \omega^{\prime}\right)$ elements. In the case of pure single electron states $\varphi_{1,2}(t)$ emitted by each source above the thermal excitations of the Fermi sea, the general expressions (1) and (2) have a simplified form and the normalized HOM noise $\Delta q=\Delta S_{33} / \Delta S_{\mathrm{HBT}}$ reads:

$$
\Delta q=1-\left|\int \varphi_{1}(t) \varphi_{2}^{*}(t) \mathrm{d} t\right|^{2} .
$$

The overlap between the states can be experimentally varied by tuning the emission delay $\tau$ between the two electron sources. For long time delays, classical random partitioning is observed: $\Delta q=1$. For short time delay, two-particle interferences occur leading to a dip in the output noise, the width of which corresponds to the duration of the emitted wavepackets. Measuring the noise suppression thus provides a quantitative measurement of the coherence of a single elementary excitation.

HOM interferometry offers several advantages compared to single particle interferometry such as Mach-Zehnder interferometry (MZI), even though it involves the measurement of current correlations instead of current. First, as noticed by Hanbury Brown and Twiss in their seminal experiment [32, intensity interferometry (such as HOM) is not sensitive to phase fluctuations. Consequently, path lengths only need to be controlled at the wavepacket scale compared to the wavelength in MZI. This has led to the success of intensity interferometry in astronomy [33] and, in the present context, enables us to escape the need for controlling the design of the interferometer down to the Fermi wavelength size (few nanometers) as required by amplitude interferometry tomography protocols [20]. More importantly, due to its extended nature (few microns), Coulomb interaction is known to occur within the MZI itself [9]. Because the experimental data cannot be corrected for the associated decoherence effects within the MZI, one cannot reconstruct the single electron coherence at the input of the interferometer from the outcoming current measurements. Moreover, 
predictions for the output coherence can even be hard to obtain. The recent experiment [10] in which energy resolved electronic excitations are continuously injected shortly before a MZI illustrates this point. When such an excitation has only been weakly affected along its propagation between its injection point and the input of the MZI, predictions for the output signals can be made [10, 34, 35] that compare favorably to the experiment. But the general case where the input state has already been strongly affected by the Coulomb interaction is much more complex and no general prediction has been obtained yet. Such problems do not occur when using the HOM interferometer as it consists of a point-like beam-splitter within which interaction effects can be neglected.

In the following, HOM interferometry is used to quantitatively analyze the decoherence of a single electronic excitation propagating along the outer edge channel of the integer quantum Hall regime at filling factor $\nu=3$ and $\nu=2$.

\section{SINGLE ELECTRON DECOHERENCE}

Figure 2 presents $\Delta q(\tau)$ at filling factor $\nu=2$ for three values of the source emission time $\tau_{e}$ which sets the temporal size of the emitted wavepackets. A dip is observed for the three curves around $\tau=0$, revealing the reduction of random partition noise by twoelectron interferences. As expected, the width of the dip increases for increasing escape time $\tau_{e}$ corresponding to increasing wavepacket width. However, none of the dips reaches the full suppression $\Delta q(0)=0$ showing that we fail to reach the collision of perfectly indistinguishable wavepackets. Defining the contrast $\gamma$ of two-particle interferences in relation with the depth of the HOM dip: $\gamma=1-\Delta q(0), \gamma$ is reduced when $\tau_{e}$ increases. Electron emission can then be modeled as a Breit-Wigner resonance between the dot and the edge channel in energy space [19]:

$$
\widetilde{\varphi}_{e}(\omega)=\frac{\mathcal{N}_{e} \Theta(\omega)}{\omega-\omega_{e}+\frac{i}{2 \tau_{e}}}
$$

where $\Theta(\omega)$ is the Heaviside step function confining the wavepacket to the accessible electronic energy levels and $\mathcal{N}_{e}$ a normalization constant. The wavepacket is parametrized by the emission energy of the electron $\omega_{e}=0.7 \mathrm{~K}$ and the resonance width $1 / \tau_{e}$. The time $\tau_{e}$

defined in this way indeed corresponds to the wavepacket duration expressed in the time 
domain [36]:

$$
\varphi_{e}(t)=\frac{\Theta(t)}{\sqrt{\tau_{e}}} e^{i \omega_{e} t} e^{-t / 2 \tau_{e}} .
$$

Without decoherence, such single electron wavepackets would lead to the following normalized HOM noise: $\Delta q(\tau)=1-e^{-|\tau| / \tau_{e}}$. To account for the observed non unit contrast $\gamma$, data are fitted (solid lines) by $\Delta q(\tau)=1-\gamma e^{-|\tau| / \tau_{e}}$. The escape times extracted from the fits correspond to $75 \pm 6$ (red trace), $110 \pm 13$ (blue trace) and $140 \pm 13$ ps (black trace). They differ, in particular for the shortest time, from the measurements of $\tau_{e}$ extracted from the phase of the a.c. current first harmonic generated by the sources[22]: $\tau_{e}=30 \pm 5,100 \pm 18$ and $180 \pm 50$ ps. The difference can be understood firstly by the non-zero rise time of the excitation pulse (typically 30 ps) and secondly by the inter-channel Coulomb interaction which leads to a widening of the current pulse [37]. Both effects are not accounted for by our independent measurement of $\tau_{e}$ from the a.c. current phase which probes the time delay between the excitation pulse and the emitted current. It is thus neither sensitive to the excitation pulse rise time nor to the charge fractionalization occurring on the outer channel [37] as the current measurement only probes the charge mode. This explains the data/fit discrepancy for short emission times. For long emission times, the relative importance of these effects decreases and the agreement with the exponential description is better. The contrast $\gamma$ extracted from the fits decreases from $0.65 \pm 0.04$ (red line) to $0.43 \pm 0.035$ (blue line) and $0.3 \pm 0.015$ (black line) showing that the indistinguishability of incoming electrons decreases with increasing escape time. On long escape times, two-electron interferences are almost fully suppressed.

\section{DECOHERENCE SCENARII}

Several possibilities could be envisioned. A first hypothesis is that the emission of undistinguishable electrons is prevented by differences between the two sources, either from sample construction or related to environmental noise leading to random fluctuations in the energy at which electrons are emitted [38]. In a second hypothesis, the contrast reduction could be related to an energy dependence of the beam-splitter reflection $R(\epsilon)$ [39]. However, according to [39], the contrast should increase with increasing wavepacket length which is opposite to what we observe. Finally, the third hypothesis relies on Coulomb induced decoherence [6, 18] along propagation as the quasiparticle gets entangled with the neighboring edge channel act- 
ing as an environment [37]. As discussed in the introduction, we shall see now that a careful analysis of the experimental data supports the Coulomb induced decoherence scenario.

Considering the first hypothesis, there are two parameters on which we can act to tune the source: the escape time $\tau_{e}$ by varying the potential barrier coupling the dot to the edge channel, and the energy $\omega_{e}$ at which electrons are emitted by changing the dot static potential. As can be seen on Fig. 3 the effect of escape time detuning $\left(\tau_{e, 1}=60 \mathrm{ps}, \tau_{e, 2}=160\right.$ ps) can easily be seen on the HOM trace. The curve $\Delta q(\tau)$ becomes asymmetric (see black trace), as predicted theoretically [36]: one side falls very quickly (source with short escape time) while the other falls much more slowly (source with long escape time). The observed contrast is very close to the one measured for $\tau_{e, 1}=\tau_{e, 2}=100 \mathrm{ps}$ (see Fig,2) corresponding roughly to the average emission time in the detuned case. Escape time asymmetries thus cannot explain the contrast reduction observed on Fig. 2. Surprisingly, no variation of the contrast is observed when the emission energies are detuned by varying the dot potential or by applying on purpose external noise to the static dot potential (see inset of Fig. 3 where a blurring of approximately $400 \mathrm{mK}$ of the emission energy of one dot is applied). The effects of energy fluctuations can be estimated theoretically by averaging HOM traces calculated using the Floquet scattering formalism [40 42] using gaussian fluctuations of amplitude $k_{b} T_{n}$ of the energy of one dot. It predicts that for $\tau_{e}=40 \mathrm{ps}$, the contrast should vary from 0.8 to 0.5 when the noise amplitude varies from $T_{n}=140 \mathrm{mK}$ to $T_{n}=400 \mathrm{mK}$. Here the value $T_{n}=400 \mathrm{mK}$ corresponds to the amplitude of the applied gate noise on Fig. 3 (insert) while $T_{n}=140 \mathrm{mK}$ is the maximum spurious gate noise compatible with the width of the conductance peaks deduced from current measurements as a function of the dot potential. On the contrary, we observe no substantial modification of the contrast when the additional noise is applied (whereas an almost complete blurring of the conductance peaks is observed). This means that our experimental data do not support the inhomogeneous broadening hypothesis.

To understand the mechanism responsible for the contrast reduction, we have plotted the variation of contrast $\gamma$ with emission time $\tau_{e}$ for filling factors 2 and 3 on Fig. 4 it falls quickly on short time both for $\nu=2$ and $\nu=3$ with faster contrast reduction for $\nu=3$ compared to $\nu=2$. To account for the contrast dependence in the escape time, let us introduce a phenomenogical coherence time $\tau_{c}$ on which the off diagonal terms of the coherence decay to zero : $\Delta \mathcal{G}^{(e)}\left(t, t^{\prime}\right) \rightarrow e^{-\left|t-t^{\prime}\right| / \tau_{c}} \Delta \mathcal{G}^{(e)}\left(t, t^{\prime}\right)$. Then, only time components $\left(t, t^{\prime}\right)$ with $\left|t-t^{\prime}\right| \leq \tau_{c}$ 
of the wavepacket can interfere on the splitter whereas $\left|t-t^{\prime}\right| \geq \tau_{c}$ components are subject to random partitioning. Using (1), this phenomenological decoherence factor predicts a reduction of the contrast related to the ratio $\tau_{c} / \tau_{e}: \gamma=\left(1+2 \tau_{e} / \tau_{c}\right)^{-1}$ where the factor 2 reflects the presence of two sources on which decoherence acts. The plain lines on Fig. 4 represent fits with the above mentioned expression $\gamma\left(\tau_{e}\right)$, providing $\tau_{c}=60$ ps at $\nu=3$ (red line) and $\tau_{c}=98 \mathrm{ps}$ at $\nu=2$ (blue line). The difference in $\tau_{c}$ between $\nu=3$ and $\nu=2$ suggests that decoherence occurs during propagation and is related to interchannel Coulomb interaction which depends on the number of co-propagating edge channels.

\section{SINGLE ELECTRON FRACTIONALIZATION: THE DEATH OF THE ELE- MENTARY QUASIPARTICLE}

Let us now review the theoretical models for Coulomb interaction effects on single electron coherence along propagation within two copropagating edge channels. Intra and inter-channel effective screened Coulomb interaction can be discussed efficiently within the bosonization 43 framework. It essentially states that all excitations of a one-dimensional chiral edge channel can be described in terms of collective bosonic modes, called edge magnetoplasmons (EMP). At $\nu=2$, strong interactions between the two channels lead to the emergence of the bosonic symmetric charge eigenmode with velocity $v_{\rho}$ and the anti-symmetric neutral or dipolar eigenmode with velocity $v_{n}$ [35]. Since its introduction, this physical picture has been directly confirmed, at least at low enough frequencies (below $10 \mathrm{GHz}$ ) through finite frequency admittance measurements [44. As the two modes propagate at different velocities, a single electronic excitation propagating on length $l$ in the outer channel splits into two pulses carrying a fractional charge [37, 45] e/2 separated by the fractionalization time $\tau_{s}=l / v_{n}-l / v_{\rho}$. This phenomenon already discussed in the context of quantum wires (1D non-chiral Luttinger liquids) [46-48] is expected to induce the disappearance of the electron as a genuine quasiparticle [5].

We now turn to the modelization of Coulomb interaction in our experiment. Following Ref.[6], we decompose the edge channel into three parts. The first is a non-interacting region located at $x=0$ in which the electron injection occurs. We assume here that, as experimentally observed [22, 41], the tunneling process from the dot is not affected by interactions apart from the renormalization of the dot parameters (electron emission in 
a strongly interacting system like the fractional quantum Hall effect deserves a different discussion [49]). The second is the interaction region $(0<x<l)$ of length $l \approx 3 \mu m$ where we describe the Coulomb interaction as a local density-density interaction term acting within each edge channel (intrachannel interaction) and between edge channels (interchannel interaction) and fully parameterized by the fractionalization time $\tau_{s}$. The third part is the beam-splitter where we assume that electrons are locally non-interacting and that Coulomb interaction does not couple together edge channels located on opposite sides of the splitter. Importantly, the chirality of the edge channels as well as the short-range nature of the interaction (which constitutes a good approximation at low enough energy [44]) allow us to extend the interaction region from infinitesimally after the point of injection to infinitesimally before the beam splitter and to neglect any back-action of the interaction region on the emission process from the quantum dot.

Single electron emission is modeled by considering that each source $\alpha=1,2$ generates in the outer edge channel the prepared state $\left|\varphi_{\alpha}, F\right\rangle=\int \mathrm{d} \omega \widetilde{\varphi}_{e, \alpha}(\omega) c^{\dagger}(\omega)|F\rangle$ where $c^{\dagger}(\omega)$ creates an electron at a given energy $\omega$ on top of the Fermi sea. Here $\widetilde{\varphi}_{e, \alpha}(\omega)$ is the single electron wavefunction generated by source $\alpha$ in the energy domain given by Eq.(5) (we assume that the hole emission process at energy $\omega_{h}=\omega_{e}$ gives the same result as the electron case). An equivalent description of the initial state can be written as a quantum superposition in real space [6, 18, 34] by extending the non interacting region for $x \leq 0$. In this description, the initial state is represented by the exponentially decaying wavepacket of Eq. (6) evaluated at $t=-x / v_{F}$. The initial wavepacket being fully located in the non-interacting region $x \leq 0$, the velocity $v_{F}$ is the non-interacting Fermi velocity.

Starting from this initial state, analytical calculations [6, 18] have been developed to compute the electronic coherence $\Delta \mathcal{G}^{(e)}\left(t, t^{\prime}\right)$ and the HOM correlations $\Delta q(\tau)$ at the output of the interaction region. Using the bosonization technique and numerical evaluation of the resulting non-perturbative expressions, we first numerically compute the excess electronic coherence function at $T=0 \mathrm{~K}$ in the Wigner representation [50] $\Delta W^{(e)}(\bar{t}, \omega)$, obtained from the Fourier transform of $\Delta \mathcal{G}^{(e)}(\bar{t}+\tilde{\tau} / 2, \bar{t}-\tilde{\tau} / 2)$ on the time difference $\tilde{\tau}$. $\Delta W^{(e)}(\bar{t}, \omega)$ is plotted on Fig 5 for increasing values of the propagation length or equivalently of $\tau_{s}$. The choice of the Wigner representation proves particularly useful here, first because it combines temporal and energetic aspects of single electron decoherence, but also because it allows for a classical limit, where $W^{(e)}(\bar{t}, \omega)$ represents the occupation probability of states at energy $\omega$ 
as a function of time $\bar{t}$. This classical interpretation fails for $W^{(e)}(\bar{t}, \omega)<0$ or $W^{(e)}(\bar{t}, \omega)>1$ [50].

Here, $\Delta W^{(e)}(\bar{t}, \omega)$ provides a direct visualization of the evolution of the single electron wavepacket under the influence of Coulomb interaction leading to the destruction of the single electron[6] as shown on Fig, F. For $\tau_{s}=0, \Delta W^{(e)}(\bar{t}, \omega)$ corresponds to the Wigner representation of the pure single electron state (6). Along the energy axis, $\Delta W^{(e)}$ is very broad on short times (as a consequence of the Heisenberg uncertainty principle) and becomes peaked around the emission energy $\omega_{e}$ on a typical timescale given by $\tau_{e}$. Ripples of negative or above unity values of $\Delta W^{(e)}(t, \omega)$ show the non-classical nature of the single electron state. After a short propagation length, $\tau_{s}=28 \mathrm{ps}$, before the fractionalization in two pulses has occurred, energy relaxes and the spectral weight at $\omega_{e}$ is transferred close to the Fermi energy $\left(\omega_{e}=0\right)$. The non-classical ripples are also almost completely washed out. On longer propagation length $\tau_{s}=70 \mathrm{ps}$, the fractionalization in two distinct pulses occurs and can be seen along the temporal axis. As two pulses of charge $e / 2$ cannot correspond to a single quasiparticle excitation of the Fermi sea, collective neutral excitations are created. This can be seen on $\Delta W^{(e)}(\bar{t}, \omega)$ by its negative values below the Fermi energy (corresponding to the creation of holes) compensated by an increase of $\Delta W^{(e)}(\bar{t}, \omega)$ above the Fermi energy (corresponding to the creation of the same number of electrons).

As can be seen on Eq.(3), the HOM dip encodes information on the overlap of single electron coherences, which can be rewritten in term of overlap between the Wigner distributions of sources 1 and 2. :

$$
\Delta S_{H O M}=4 R(1-R) e^{2} \int \frac{d \omega}{2 \pi} \overline{\Delta W_{1}^{(e)}(\bar{t}, \omega) \Delta W_{2}^{(e)}(\bar{t}, \omega)} \bar{t}
$$

The single electron decoherence scenario represented by the evolution of the Wigner distribution $\Delta W^{(e)}(\bar{t}, \omega)$ as a function of propagation length on Fig. 5 can thus be tested by means of HOM data versus theory comparison[6, 18].

The upper left panel of Fig.6 presents the data of the HOM traces $\Delta q(\tau)$ for various emission times $\tau_{e}$ together with theoretical predictions at $T=0$ and $T=100 \mathrm{mK}$ providing an evaluation of the effect of finite temperature on single electron decoherence. The interaction parameter is set to $\tau_{s}=70 \mathrm{ps}$ which is extracted from high frequency admittance measurements [44] performed on a similar sample coming from the same batch and which confirmed the validity of the short range interaction model up to frequencies $f \leq 6 \mathrm{GHz}$. 
The parameter $\tau_{s}=70 \mathrm{ps}$ has also been successfully used to describe the charge fractionalization in Ref. [37] using the same sample as in the present work (at the same value of the magnetic field). The red, blue, and black curves represent these theoretical predictions taking $\tau_{e}=34,91$ and 147 ps. These values agree within experimental resolution with the values of $\tau_{e}$ extracted from the measurements of the average current. In particular for the short time $\tau_{e}=34 \mathrm{ps}$, theoretical predictions capture the broadening of the electronic wavepacket by the fractionalization process which leads to an overestimate by a factor 2 of the emission time extracted from the exponential fit of the dip (although the experimental resolution is not good enough to observe the predicted side peaks for $\tau_{e}=34 \mathrm{ps}, T=0.1$ $\mathrm{K})$. The agreement between the data and the predictions is good: once the width of the dip has been chosen to match the data, the values of the contrast also agree. Note that contrary to Fig 2 where a phenomenogical description of decoherence involving two adjustable parameters was used, we are able here to fit the full HOM trace using only experimentally measured parameters (emission time $\tau_{e}$ and interaction strength $\tau_{s}$ ). The differences between the calculated HOM curves at different temperatures are small showing a small influence of temperature on single electron decoherence. This is explained by the electron emission energy $\hbar \omega_{e}>k_{b} T_{e}$.

The lower left panel of Fig 6 presents the data-model comparison when the emission times of the two sources are detuned. The agreement is also very good providing the right value for the contrast of two-particle interferences contrary to the non-interacting predictions (black blurred line).

Last but not least, the lower right panel of Fig 6 exhibits the most striking distinctive prediction of the interaction model: the contrast and shape of the HOM trace is almost unchanged when the emission energy of one of the two sources is varied (from $0.7 \mathrm{~K}$ to 0.3 $\mathrm{K})$. This behavior is completely different from the non-interacting model predictions (black and red blurred lines) for which the contrast varies strongly from 1 to 0.25 when the energies are detuned by $400 \mathrm{mK}$ at $\tau_{e}=40 \mathrm{ps}$. Surprisingly, in the detuned case, interactions lead to enhancement of the contrast compared to the non-interacting prediction. This restauration of indistinguishability by decoherence is a consequence of electronic relaxation. At a quantitative level, it can be shown that at long times, the resulting single electron coherence only depends on the shape of the initial current pulse (here encoded in the duration $\tau_{e}$ ) and of the propagation distance but not anymore of its initial injection energy. This erasure effect 
is a consequence of the entanglement of the electronic degrees of freedom of the outer edge channel where the single electron excitation is injected with the inner one [6]. Quantitatively confirming this effect is a strong signature of the single electron decoherence scenario described within the bosonization framework.

Finally, the data model comparison of the $\tau_{e}$ dependence of the contrast $\gamma$ can be seen on the upper right panel of Fig 6 giving a coherence time $\tau_{c}=86 \mathrm{ps}(T=100 \mathrm{mK})$ and $\tau_{c}=104$ ps $(T=0 \mathrm{~K})$ close to the data $\left(\tau_{c}=98\right.$ ps at $\left.\nu=2\right)$. The overall agreement is good even if, for long escape times, the data tends to accumulate above the theoretical predictions. However, for $\tau_{e} \geq 180 \mathrm{ps}, \tau_{e}$ cannot be neglected compared to the drive half-period and the probability $P$ of single charge emission starts to decrease $(P \leq 0.9)$ [51, 52]. The comparison with perfect single electron states thus ceases to be valid in this long emission time limit.

\section{CONCLUSION}

To conclude, we have analyzed the coherence of single electron states propagating along a one-dimensional edge channel using HOM interferometry. We observe a strong reduction of the HOM contrast when the width of the emitted single electron wavepackets is increased from which a coherence time $\tau_{c} \approx 100 \mathrm{ps}$ (at $\nu=2$ ) can be extracted. Our results are in quantitative agreement with the Coulomb interaction induced decoherence along propagation providing the first direct evidence of the destruction scenario of a single quasiparticle in a one dimensional conductor. The outcome of this study extends, beyond charge propagation in conductors, to a large variety of low-dimensional systems where the Luttinger paradigm is relevant [53/55]. For example, those decoherence scenarii could be studied as a function of interaction strength in low dimensional cold atomic systems where single elementary excitations can now be manipulated [56] and experimental resolutions now reach the single atom scale [57].

\section{ACKNOWLEDGMENTS}

This work is supported by the ERC consolidator grant 'EQuO' and the ANR grant '1shot reloaded', ANR-14-CE32-0017. The development of the HEMTs used for cryogenic readout electronics in this experiment was supported in part by the European FP7 space project 
CESAR grant No. 263455.

[1] F. Pierre, A.B. Gougam, A. Anthore, H. Pothier, D. Esteve, and N. O. Birge, Dephasing of electrons in mesoscopic metal wires, Phys. Rev. B 68, 085413 (2003).

[2] D. Pines and P. Nozieres, The Theory of Quantum Liquids, Vol. I, W. A. Benjamin , Inc., New York (1966).

[3] J. M. Luttinger, An Exactly Soluble Model of a Many-Fermion System, J. Math. Phys. 4, 1154 (1963).

[4] O. M. Auslaender, H. Steinberg, A. Yacoby,Y. Tserkovnyak, B. I. Halperin, K. W. Baldwin, L. N. Pfeiffer, K. W. West, Spin-Charge Separation and Localization in One Dimension, Science 308, $88(2005)$.

[5] K. LeHur, Electron fractionalization-induced dephasing in Luttinger liquids, Phys. Rev. B 65, $233314(2002)$.

[6] D. Ferraro, B. Roussel, C. Cabart, E. Thibierge, G. Fève, C. Grenier, and P. Degiovanni, RealTime Decoherence of Landau and Levitov Quasiparticles in Quantum Hall Edge Channels, Phys. Rev. Lett. 113, 166403 (2014).

[7] J.D.M Haldane, Luttinger liquid theory of one-dimensional quantum fluids: I. Properties of the Luttinger model and their extension to the general 1D interacting spinless Fermi gas, J. Phys. C: Solid State Phys., 14, 2585 (1981).

[8] I. Neder, M. Heiblum,Y. Levinson, D. Mahalu, and V. Umansky, Unexpected Behavior in a Two-Path Electron Interferometer, Phys. Rev. Lett. 96, 016804 (2006).

[9] P. Roulleau, F. Portier, D. C. Glattli, P. Roche, A. Cavanna, G. Faini, U. Gennser, and D. Mailly, Direct Measurement of the Coherence Length of Edge States in the Integer Quantum Hall Regime, Phys. Rev. Lett. 100, 126802 (2008).

[10] S. Tewari, P. Roulleau, C. Grenier, F. Portier, A. Cavanna, U. Gennser, D. Mailly, and P. Roche, Robust quantum coherence above the Fermi sea, Phys. Rev. B 93, 035420 (2016).

[11] D.T. McClure, Y. Zhang, B. Rosenow, E.M. Levenson-Falk, C.M. Marcus, L.N. Pfeiffer, and K. W. West, Edge-State Velocity and Coherence in a Quantum Hall Fabry-Prot Interferometer, Phys. Rev. Lett. 103, 206806 (2009). 
[12] Y. Ji, Y. Chung, D. Sprinzak, M. Heiblum, D. Mahalu, and H. Shtrikman, An electronic MachZehnder interferometer, Nature, 422, 415-418 (2003).

[13] C. Altimiras, H. le Sueur, U. Gennser, A. Cavanna, D. Mailly, and F. Pierre, Non-equilibrium edge-channel spectroscopy in the integer quantum Hall regime, Nat. Phys. 6, 34 (2010).

[14] H. LeSueur, C. Altimiras, U. Gennser, A. Cavanna, D. Mailly, and F. Pierre, Energy Relaxation in the Integer Quantum Hall Regime, Phys. Rev. Lett. 105, 056803 (2010).

[15] S. Ol'khovskaya, J. Splettstoesser, M. Moskalets, and M. Büttiker, Shot Noise of a Mesoscopic Two-Particle Collider, Phys. Rev. Lett. 101, 166802 (2008).

[16] E. Bocquillon, V. Freulon, J.-M Berroir, P. Degiovanni, B. Plaçais, A. Cavanna, Y. Jin, and G. Fève, Coherence and Indistinguishability of Single Electrons Emitted by Independent Sources, Science 339, 1054 (2013).

[17] C. K. Hong, Z. Y. Ou, and L.Mandel, Measurement of subpicosecond time intervals between two photons by interference, Phys. Rev. Lett. 59, 2044 (1987).

[18] C. Wahl, J. Rech, T. Jonckheere, and T. Martin, Interactions and Charge Fractionalization in an Electronic Hong-Ou-Mandel Interferometer, Phys. Rev. Lett. 112, 046802 (2014).

[19] C. Grenier, R. Hervé, E. Bocquillon, F. D. Parmentier, B. Plaçais, J.-M. Berroir, G. Fève, and P. Degiovanni, Single electron quantum tomography in quantum Hall edge channels, New Journal of Physics 13, 093007 (2011).

[20] G. Haack, M. Moskalets, J. Splettstoesser and M. Büttiker, Coherence of single-electron sources from Mach-Zehnder interferometry, Phys. Rev. B 84, 081303 (2011).

[21] E. Bocquillon, V. Freulon, F. D. Parmentier, J.-M. Berroir, B. Plaçais, C. Wahl, J. Rech, T. Jonckheere, T. Martin, C. Grenier, D. Ferraro, P. Degiovanni, and G. Fève, Electron quantum optics in ballistic chiral conductors, Annalen der Physik 526, 1 (2014).

[22] G. Fève, A. Mahé, J.-M. Berroir, T. Kontos, B. Plaçais, D. C. Glattli, A. Cavanna, B. Etienne, Y. Jin, An On-Demand Coherent Single Electron Source, Science 316, 1169 (2007).

[23] J.D. Fletcher, P. See, H. Howe, M. Pepper, S.P. Giblin, J. P. Griffiths, G.A.C. Jones, I. Farrer, D.A. Ritchie, T.J.B.M. Janssen, and M. Kataoka, Clock-Controlled Emission of SingleElectron Wave Packets in a Solid-State Circuit, Phys. Rev. Lett. 111, 216807 (2013).

[24] J. Dubois, T. Jullien, F. Portier, P. Roche, A. Cavanna, Y. Jin, W. Wegscheider, P. Roulleau, and D. C. Glattli, Minimal-excitation states for electron quantum optics using levitons, Nature 502, 659 (2013). 
[25] N. Ubbelohde, F. Hohls, V. Kashcheyevs, T. Wagner, L. Fricke, B. Kästner, K. Pierz, H. W. Schumacher, and R. J. Haug, Partitioning of on-demand electron pairs, Nature Nanotechnology 10, 46-49 (2015).

[26] M. Kataoka, N. Johnson, C. Emary, P. See, J. P. Griffiths, G. A. C. Jones, I. Farrer, D. A. Ritchie, M. Pepper, and T. J. B. M. Janssen, Time-of-Flight Measurements of Single-Electron Wave Packets in Quantum Hall Edge States, Physical Review Letters 116, 126803 (2016).

[27] T. Jullien, P. Roulleau, B. Roche, A. Cavanna, Y. Jin, and D. C. Glattli, Quantum tomography of an electron, Nature 514, 603 (2014).

[28] R.C. Liu, B. Odom, Y. Yamamoto and S. Tarucha, Quantum interference in electron collision, Nature 391, 263 (1997).

[29] P. Samuelsson, E.V. Sukhorukov and M. Büttiker, Two-Particle Aharonov-Bohm Effect and Entanglement in the Electronic Hanbury BrownTwiss Setup, Phys. Rev. Lett. 92, 026805 (2004).

[30] I. Neder, N. Ofek, Y. Chung, M. Heiblum, D. Mahalu, and V. Umansky, Interference between two indistinguishable electrons from independent sources, Nature 448, 333 (2007).

[31] E. Bocquillon, F.D. Parmentier, Ch. Grenier, J.-M. Berroir, P. Degiovanni, D.C. Glattli, B. Plaçais, A. Cavanna, Y. Jin, and G. Fève, Electron quantum optics: partitioning electrons one by one, Phys. Rev. Lett. 108, 196803 (2012).

[32] R. Hanbury Brown and R. Twiss, Correlation between Photons in two Coherent Beams of Light, Nature 177, 27 (1956).

[33] R. Hanbury Brown and R. Twiss, A test of a new type of stellar interferometer on Sirius, Nature 178, 1046 (1956).

[34] A. O. Slobodeniuk, E. G. Idrisov, and E. V. Sukhorukov, Relaxation of an electron wave packet at the quantum Hall edge at filling factor $\nu=2$, Physical Review B 93, 035421 (2016).

[35] I.P. Levkivskyi and E.V. Sukhorukov, Dephasing in the electronic Mach-Zehnder interferometer at filling factor $\nu=2$, Phys. Rev. B 78, 045322 (2008).

[36] T. Jonckheere, J. Rech, C. Wahl and T. Martin, Electron and hole Hong-Ou-Mandel interferometry, Phys. Rev. B 86, 125425 (2012).

[37] V. Freulon, A. Marguerite, J.-M. Berroir, B. Plaçais, A. Cavanna, Y. Jin, and G. Fève, HongOu-Mandel experiment for temporal investigation of single-electron fractionalization, Nat. Comm. 6, 6854 (2015). 
[38] E. Iyoda, T. Kato, K. Koshino, T. Martin, Dephasing in single-electron generation due to environmental noise probed by Hong-Ou-Mandel interferometry, Phys. Rev. B 89, 205318 (2014).

[39] D. Marian, E. Colomés and X. Oriols, Time-dependent exchange and tunneling: detection at the same place of two electrons emitted simultaneously from different sources, J. Phys.: Condens. Matter 27245302 (2015).

[40] M. Moskalets, and M. Büttiker, Floquet scattering theory of quantum pumps, Phys. Rev. B 66205320 (2002).

[41] F. D. Parmentier, E. Bocquillon, J.-M. Berroir, D. C. Glattli, B. Plaçais, G. Fève, M. Albert, C. Flindt, and M. Büttiker, Current noise spectrum of a single-particle emitter: Theory and experiment, Phys. Rev. B 85165438 (2012).

[42] M. Moskalets, G. Haack and M. Büttiker, Single-electron source: Adiabatic versus nonadiabatic emission, Phys. Rev. B 87125429 (2013).

[43] J. von Delft, H. Schoeller, Bosonization for beginners-refermionization for experts, Annalen der Physik 7225 (1998).

[44] E. Bocquillon, V. Freulon, J-.M Berroir, P. Degiovanni, B. Plaçais, A. Cavanna, Y. Jin, and G. Fève, Separation of neutral and charge modes in one-dimensional chiral edge channels, Nat. Comm. 4, 1839 (2013).

[45] E. Berg, Y. Oreg, E.A. Kim, and F. vonOppen, Fractional Charges on an Integer Quantum Hall Edge, Phys. Rev. Lett. 102, 236402 (2009).

[46] I. Safi and H. Schulz, Transport in an inhomogeneous interacting one-dimensional system, Phys. Rev. B 52, R17040 (1995).

[47] H. Steinberg, G. Barak, A. Yacoby, and L. N. Pfeiffer, Charge fractionalization in quantum wires, Nat. Phys. 4, 116(2007).

[48] H. Kamata, N. Kumada, M. Hashisaka, K. Muraki, and T. Fujisawa, Fractionalized wave packets from an artificial Tomonaga Luttinger liquid, Nat. Nanotech. 9, 177 (2014).

[49] D. Ferraro, J. Rech, T. Jonckheere, and T. Martin, Single quasiparticle and electron nanoemitter in the fractional quantum Hall regime, Phys. Rev. B 91, 205409 (2015).

[50] D. Ferraro, A. Feller, A. Ghibaudo, E. Thibierge, E. Bocquillon, G. Fève, C. Grenier, and P. Degiovanni, Wigner function approach to single electron coherence in quantum Hall edge channels, Phys. Rev. B 88, 205303 (2013). 
[51] A. Mahé, F. D. Parmentier, E. Bocquillon, J.-M. Berroir, D. C. Glattli, T. Kontos, B. Plaçais, G. Fève, A. Cavanna, and Y. Jin, Current correlations of an on-demand single-electron emitter, Phys. Rev. B 82201309 (2010).

[52] M. Albert, C. Flindt, and M. Büttiker, Accuracy of the quantum capacitor as a single-electron source, Phys. Rev. B 82041407 (2010).

[53] C. Kollath, U. Schollwöck, and W. Zwerger, Spin-Charge Separation in Cold Fermi Gases: A Real Time Analysis, Physical Review Letters 95, 176401 (2005).

[54] D. G. Angelakis, M. Huo, E. Kyoseva and L. C. Kwek, Luttinger Liquid of Photons and Spin-Charge Separation in Hollow-Core Fibers, Physical Review Letters 106, 153601 (2011).

[55] P. Jurcevic, P. Hauke, C. Maier, C. Hempel, B.P. Lanyon, R. Blatt, and C.F. Roos, Spectroscopy of interacting particles in trapped ions, Physical Review Letters 115, 100501 (2015).

[56] T. Fukuhara, A. Kantian, M. Endres, M. Cheneau, P. Schau, S. Hild, D. Bellem, U. Schollwöck, T. Giamarchi, C. Gross, I. Bloch, and S. Kuhr, Quantum dynamics of a mobile spin impurity, Nat. Phys. 9, 235 (2013).

[57] E. Haller, J. Hudson, A. Kelly, D. A. Cotta, B. Peaudecerf, G. D. Bruce, and S. Kuhr, Singleatom imaging of fermions in a quantum-gas microscope, Nat. Phys 11, 738 (2015). 


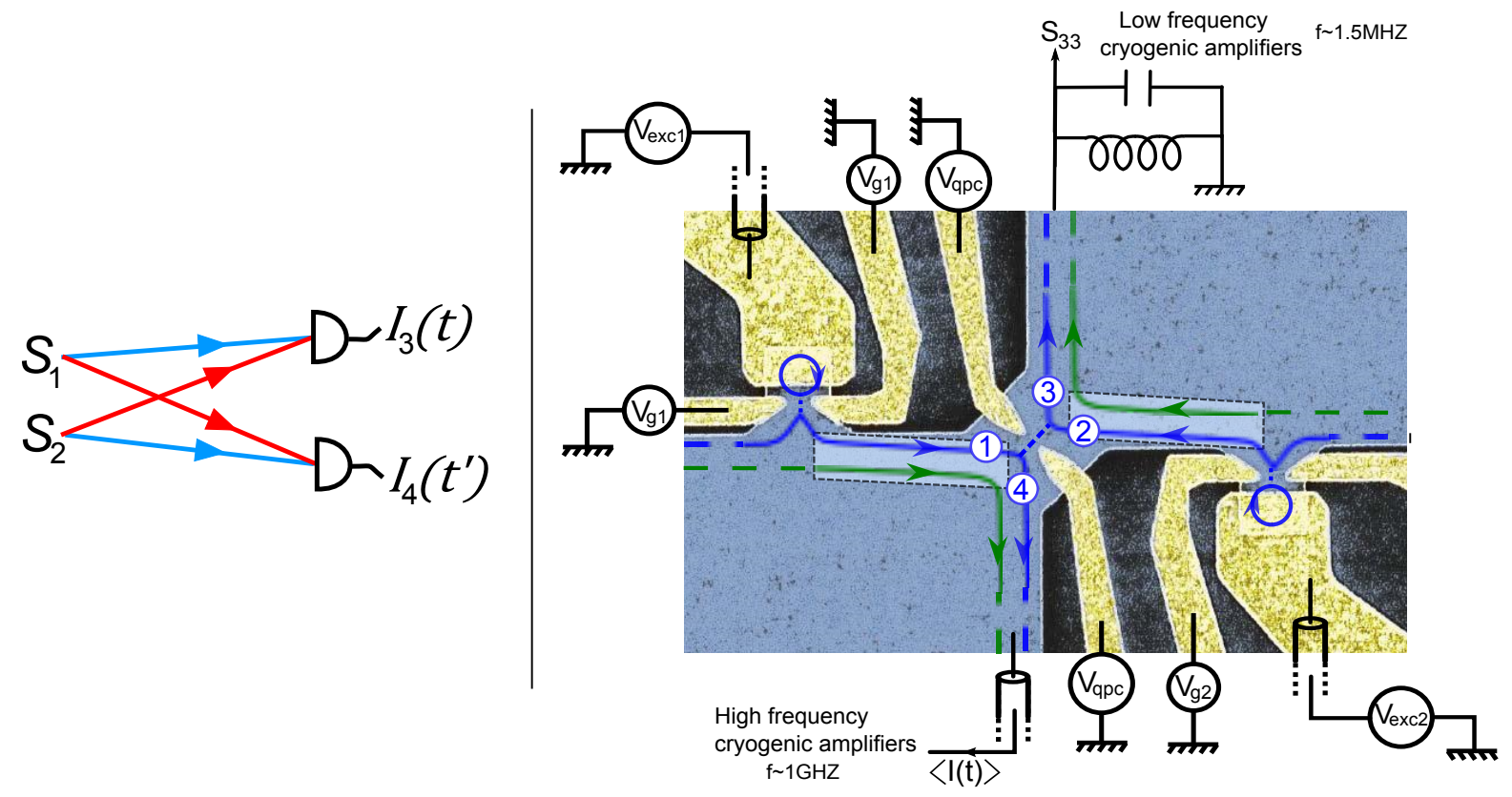

FIG. 1. Hong-Ou-Mandel interferometry Left panel, sketch of two-particle interferences. Right panel, modified scanning electronic microscope picture of the sample. The electron gas is represented in blue, the edge channels by blue (outer channel) and green (inner channel) lines, metallic gates are in gold. The emitters are placed at inputs 1 and 2 of the QPC used as an electronic beam-splitter. Single electron emission by source $i$ on the outer channel is triggered by the square voltage $V_{\mathrm{exc}, i}$ of amplitude $0.7 \mathrm{~K}$. The dot to edge transmission of source $i$ is tuned by the gate voltage $V_{\mathrm{g}, i}$. The central QPC is set to partition $(R=0.5)$ the outer channel using the gate voltage $V_{\mathrm{qpc}}$. Interaction regions of length $l \approx 3 \mu m$ are represented by light blue boxes. Average ac current measurements are performed on the splitter output 4 in order to characterize the source parameters (in particular $\tau_{e}$ ). Low frequency noise measurements $\Delta S_{33}$ are performed on output 3 . 


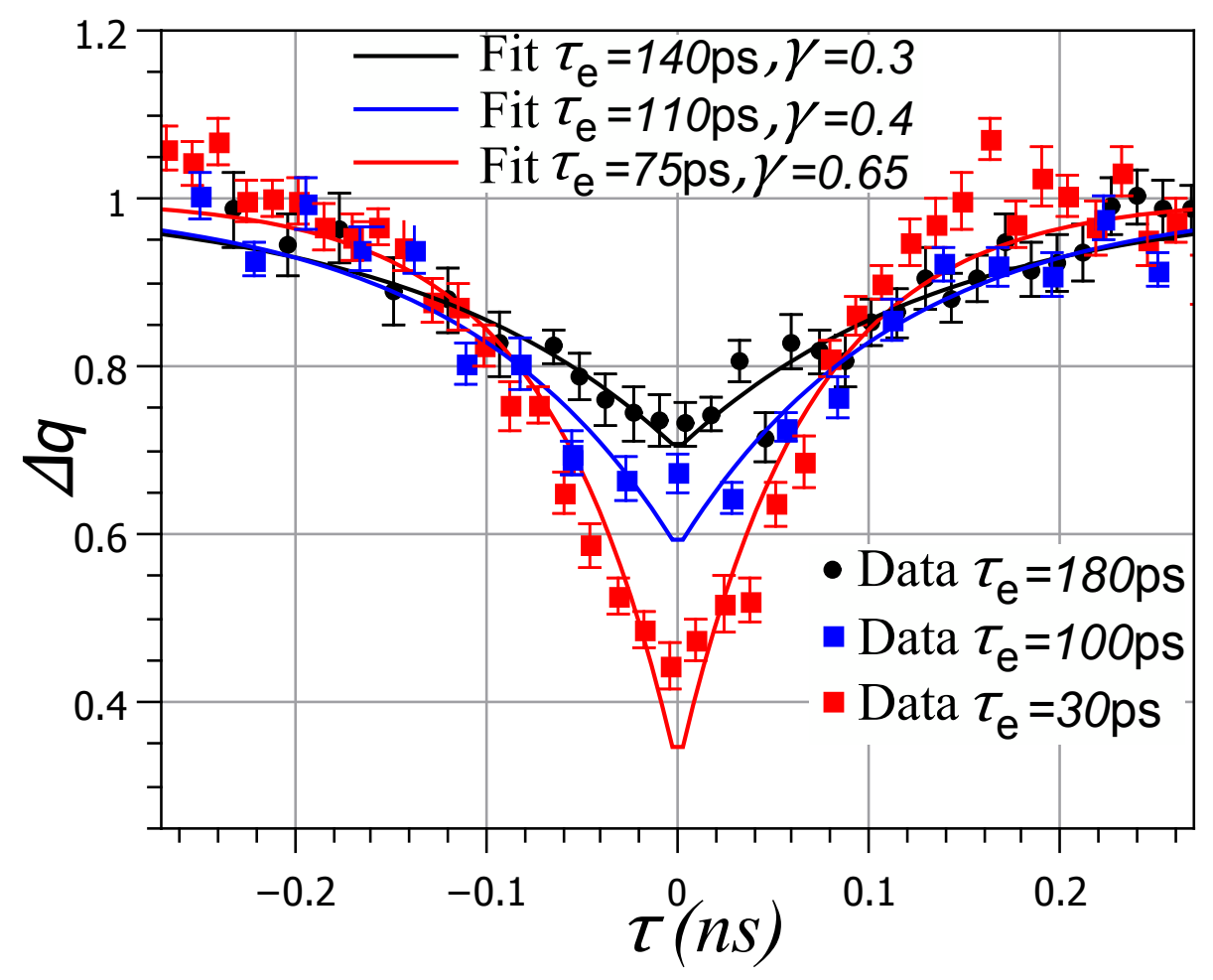

FIG. 2. Electronic Hong-Ou-Mandel experiment. HOM trace $\Delta q$ as a function of the time delay between the sources $\tau$ for three values of the emission time $\tau_{e}=30$ ps (red squares), $\tau_{e}=100 \mathrm{ps}$ (blue squares) and $\tau_{e}=180 \mathrm{ps}$ (black dots). The plain lines represent exponential fits, $\Delta q(\tau)=1-\gamma e^{-|\tau| / \tau_{e}}$. 


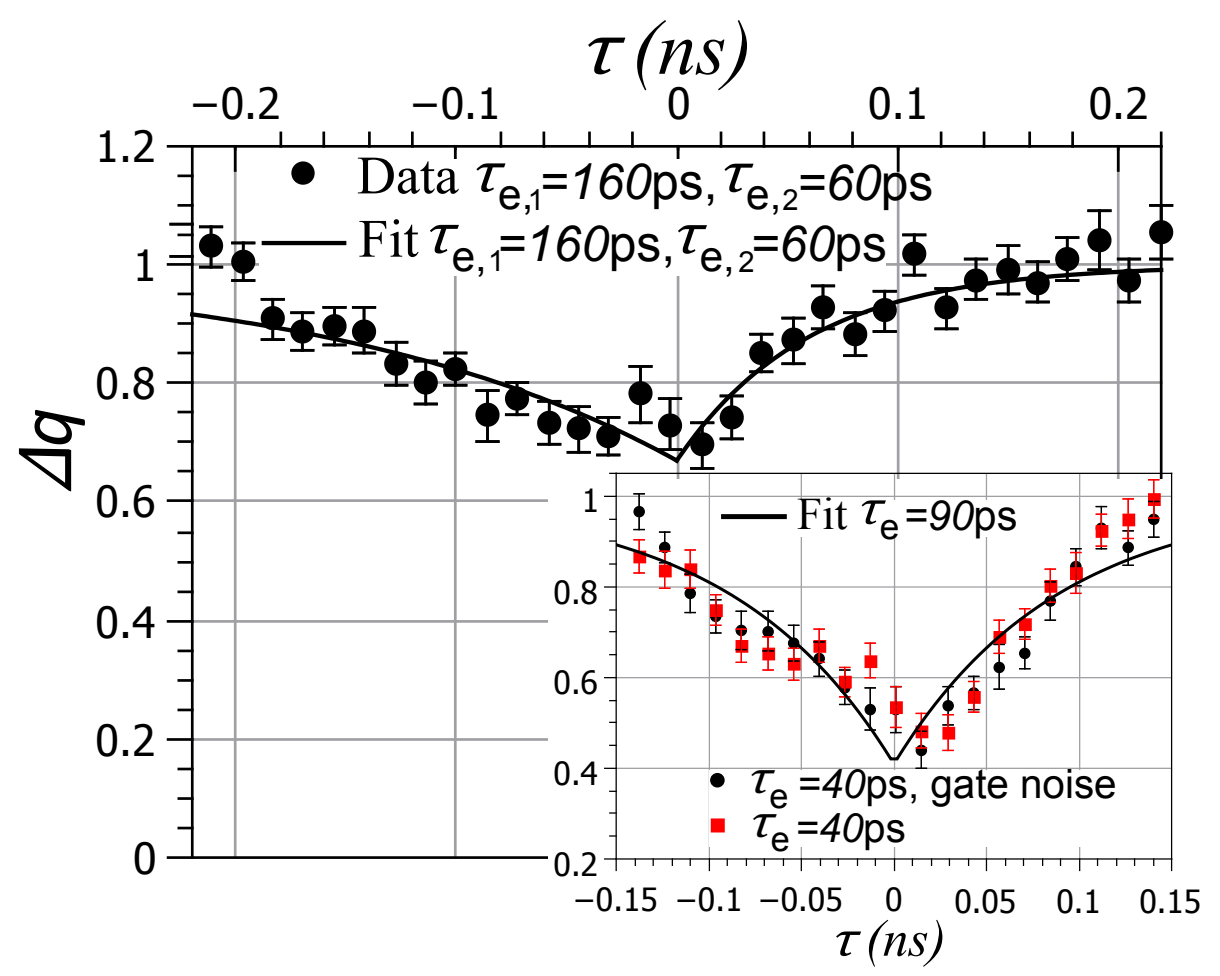

FIG. 3. Escape time asymmetry and energy emission fluctuations. $\Delta q(\tau)$ for asymmetric $\left(\tau_{1}=160, \tau_{2}=60 \mathrm{ps}\right.$, black dots) escape times. The black line is an exponential fit with $\tau_{e}=160 \mathrm{ps}$ for $\tau \leq 0, \tau_{e}=60 \mathrm{ps}$ for $\tau \geq 0$. Inset, $\Delta q(\tau)$ with (black dots) and without (red squares) external noise applied on the static potential of dot 1 . The noise amplitude corresponds to a blurring of a $400 \mathrm{mK}$ of the dot emission energy. The black line is a fit with an exponential decay. 


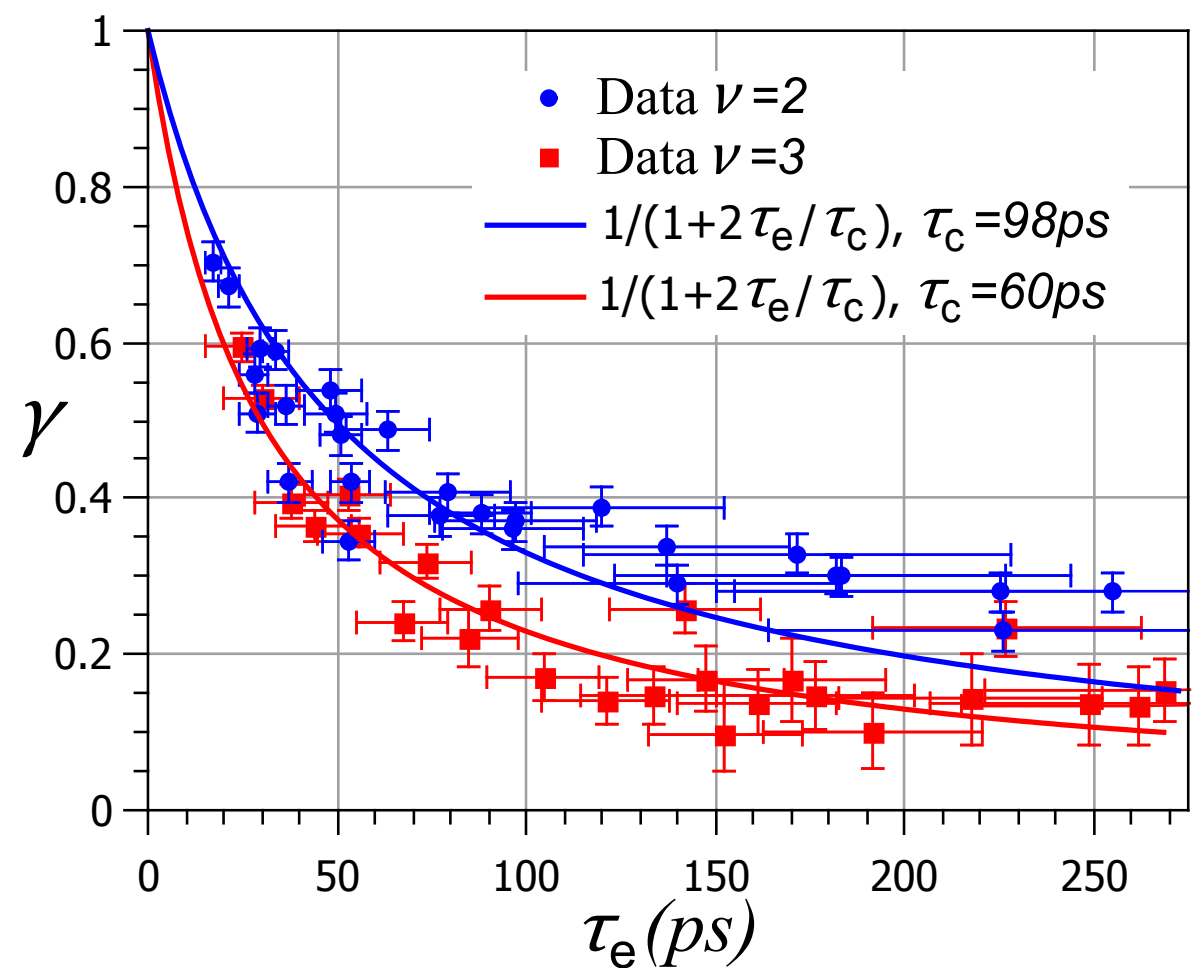

FIG. 4. Contrast versus emission time. Evolution of contrast $\gamma$ as a function of emission time $\tau_{e}$ for $\nu=2$ (blue dots) and $\nu=3$ (red squares). The plain lines correspond to the fits by the phenomenogical model $\gamma\left(\tau_{e}\right)=1 /\left(1+2 \tau_{e} / \tau_{c}\right)$. 


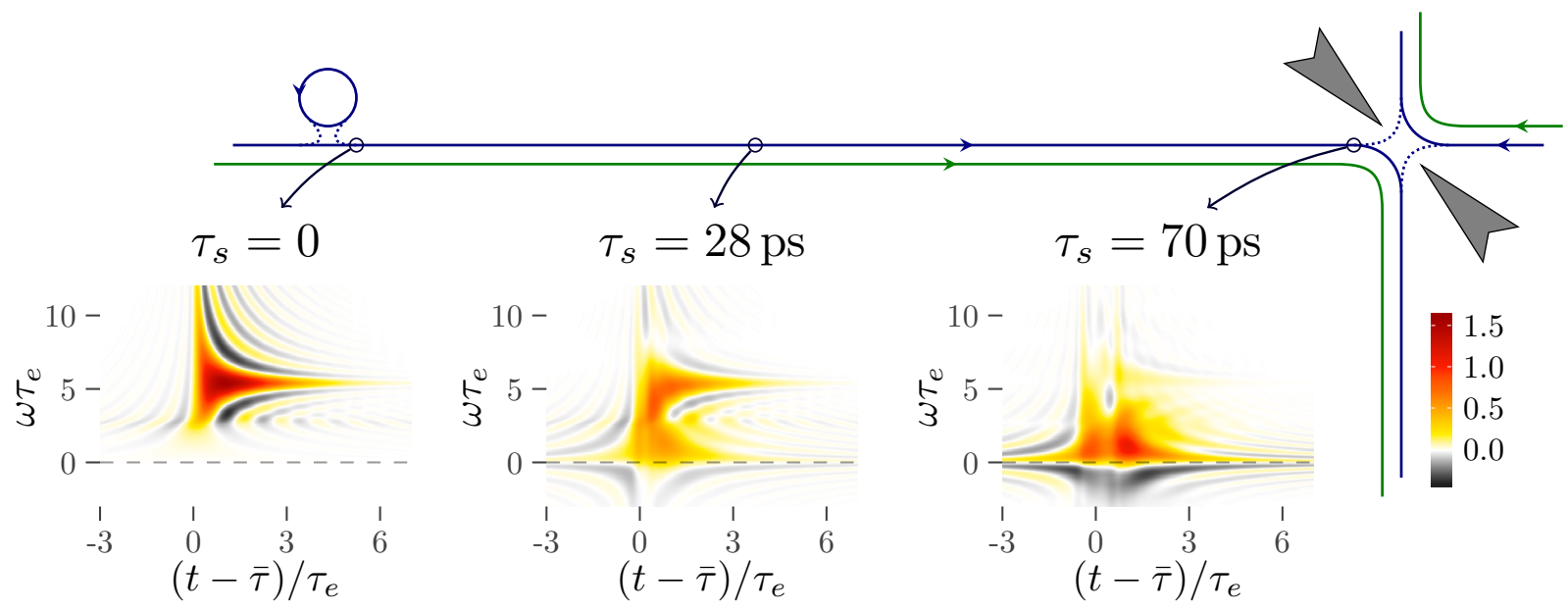

FIG. 5. Destruction of the elementary quasiparticle. Wigner representations $\Delta W^{(e)}(\bar{t}, \omega)$ of the excess single electron coherence at $T=0 \mathrm{~K}$ for different propagation lengths $\tau_{s}=0,28$ and $70 \mathrm{ps}$. The time axis are shifted by time $\bar{\tau}=l / v_{\rho}$ to account for the propagation time on length $l$. For $\tau_{s}=0, \Delta W^{(e)}(\bar{t}, \omega)$ represents the state emitted in the outer edge channel (blue line) described by eq. (6), with $\omega_{e}=0.7 \mathrm{~K}$ and $\tau_{e}=60$ ps. For $\tau_{s}=28$ and 70 ps, short range Coulomb interactions between the outer and inner (green line) edge channels are taken into account. 


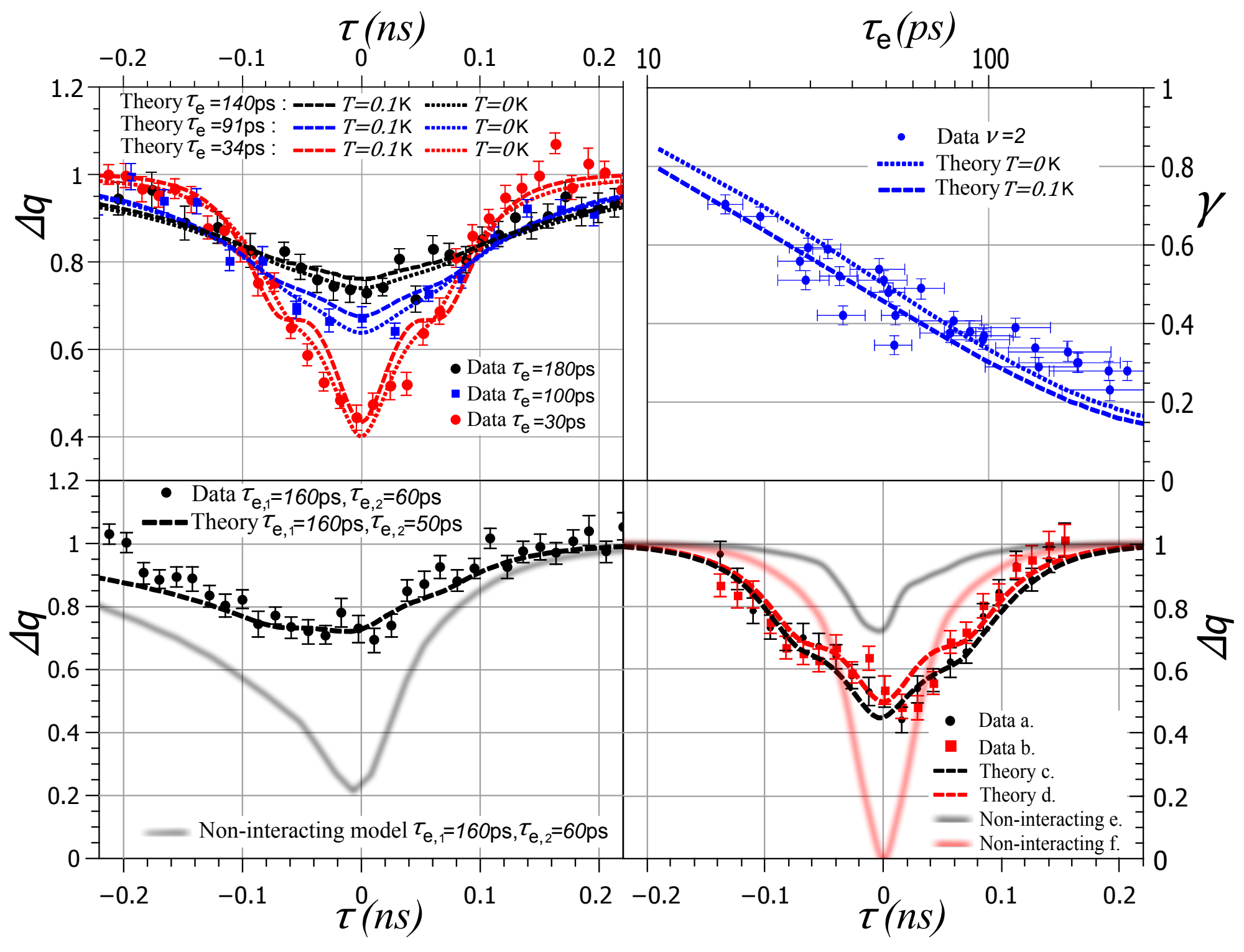

FIG. 6. Data/model comparison. Upper left panel, $\Delta q(\tau)$ for various emission times. Theory accounting for Coulomb interaction is represented in dotted line $(T=0 \mathrm{~K})$ and dashed line $(T=0.1$ $\mathrm{K})$. Lower left panel, $\Delta q(\tau)$ for asymmetric emission times. Theory predictions accounting for Coulomb interaction $(T=0.1 \mathrm{~K})$ are represented in dashed lines. Predictions of the non-interacting model in blurred black. Upper right panel, contrast $\gamma$ versus emission time $\tau_{e}$ (in log-linear scale). The dotted $(T=0)$ and dashed $(T=100 \mathrm{mK})$ lines represent theory predictions accounting for Coulomb interaction. Lower right panel, a. Data, $\tau_{e}=40 \mathrm{ps}, 400 \mathrm{mK}$ gate noise on dot 1 . b. Data, $\tau_{e}=40$ ps without gate noise. c. Theory, $T=0.1 \mathrm{~K}, \omega_{1}=0.7 \mathrm{~K}, \omega_{2}=0.3 \mathrm{~K}, \tau_{e}=40$ ps. d. Theory, $T=0.1 \mathrm{~K}, \omega_{1}=\omega_{2}=0.7 \mathrm{~K}$ e. Non-interacting model, $\omega_{1}=0.7 \mathrm{~K}, \omega_{2}=0.3 \mathrm{~K}, \tau_{e}=40$ ps. f. Non-interacting model, $\omega_{1}=\omega_{2}=0.7 \mathrm{~K}, \tau_{e}=40 \mathrm{ps}$. 